\title{
Solution of Differential Equations with the Aid of an Analytic Continuation of Laplace Transform
}

\author{
Tohru Morita ${ }^{1}$, Ken-ichi Sato ${ }^{2}$ \\ ${ }^{1}$ Tohoku University, Sendai, Japan \\ ${ }^{2}$ College of Engineering, Nihon University, Koriyama, Japan \\ Email: senmm@jcom.home.ne.jp
}

Received 4 February 2014; revised 4 March 2014; accepted 11 March 2014

Copyright (C) 2014 by authors and Scientific Research Publishing Inc.

This work is licensed under the Creative Commons Attribution International License (CC BY). http://creativecommons.org/licenses/by/4.0/

(c) (i) Open Access

\begin{abstract}
We discuss the solution of Laplace's differential equation and a fractional differential equation of that type, by using analytic continuations of Riemann-Liouville fractional derivative and of Laplace transform. We show that the solutions, which are obtained by using operational calculus in the framework of distribution theory in our preceding papers, are obtained also by the present method.
\end{abstract}

\section{Keywords}

Laplace's Differential Equation, Kummer's Differential Equation, Fractional Differential Equation, Laplace Transform, Analytic Continuation via Hankel's Contour

\section{Introduction}

Yosida [1] [2] discussed the solution of Laplace's differential equation, which is a linear differential equation, with coefficients which are linear functions of the variable. In recent papers [3] [4], we discussed the solution of that equation, and fractional differential equation of that type. The differential equations are expressed as

$$
\left(a_{2} t+b_{2}\right) \cdot{ }_{0} D_{R}^{2 \sigma} u(t)+\left(a_{1} t+b_{1}\right) \cdot{ }_{0} D_{R}^{\sigma} u(t)+\left(a_{0} t+b_{0}\right) u(t)=f(t), \quad t>0,
$$

for $\sigma=1$ and $\sigma=1 / 2$. Here $a_{l}, b_{l}$ for $l=0,1,2$ are constants, and ${ }_{0} D_{R}^{l \sigma} u(t)$ are the Riemann-Liouville fractional derivatives to be defined in Section 2 .

We use $\mathbb{R}, \mathbb{Z}$ and $\mathbb{C}$, to denote the sets of all real numbers, of all integers and of all complex numbers. We also use $\mathbb{R}_{>a}:=\{x \in \mathbb{R} \mid x>a\}, \mathbb{Z}_{>b}:=\{n \in \mathbb{Z} \mid n>b\}, \mathbb{Z}_{<c}:=\{n \in \mathbb{Z} \mid n<c\}$ and 
$\mathbb{Z}_{[b, c]}:=\{n \in \mathbb{Z} \mid b \leq n \leq c\}$ for $a \in \mathbb{R}$ and $b, c \in \mathbb{Z}$ satisfying $b<c$. If $n \in \mathbb{Z}_{>0},{ }_{0} D_{R}^{n} u(t)=\frac{\mathrm{d}^{n}}{\mathrm{~d} t^{n}} u(t)$, and ${ }_{0} D_{R}^{0} u(t)=u(t)$. We use $\lceil x\rceil$ for $x \in \mathbb{R}$, to denote the least integer that is not less than $x$. In the present paper, the variable $t$ is always assumed to take values on $\mathbb{R}_{>0}$.

Yosida [1] [2] studied the Equation (1.1) for $\sigma=1$ with $f(t)=0$, by using Mikusiński's operational calculus [5]. In [3] [4], operational calculus in terms of distribution theory is used, which was developed for the initial-value problem of fractional differential equation with constant coefficients in our preceding papers [6] [7]. In [3], the derivative is the ordinary Riemann-Liouville fractional derivative, so that the fractional derivative of a function $u(t)$ exists only when $u(t)$ is locally integrable on $\mathbb{R}_{>0}$, and the integral $\int_{0}^{1} u(t) \mathrm{d} t$ converges.

Practically, we adopt Condition B in [3], which is

Condition 1. $u(t)$ and $f(t)$ in (1) are expressed as a linear combination of $g_{v}(t)$ for $v \in \mathbb{R}_{>0}$.

Here $g_{v}(t)$ is defined by

$$
g_{v}(t)=\frac{1}{\Gamma(v)} t^{v-1}, \quad t>0
$$

for $v \in \mathbb{R} \backslash \mathbb{Z}_{<1}$, where $\Gamma(v)$ is the gamma function.

We then express $u(t)$ as follows:

$$
u(t)=\sum_{v \in S_{1}} u_{v-1} g_{v}(t),
$$

where $u_{v-1} \in \mathbb{R}$ are constants, and $S_{1}$ is a set of $v \in \mathbb{R}_{>0}$.

In a recent review [8], we discussed the analytic continuations of fractional derivative, where an analytic continuation of Riemann-Liouville fractional derivative of function $u(t)$ is such that the fractional derivative exists when $u(t)$ is locally integrable on $\mathbb{R}_{>0}$, even when the integral $\int_{0}^{1} u(t) \mathrm{d} t$ diverges.

In [4], we adopted this analytic continuation of Riemann-Liouville fractional derivative, and the following condition, in place of Condition 1.

Condition 2. $u(t)$ and $f(t)$ in (1.1) are expressed as a linear combination of $g_{v}(t)$ for $v \in S$, where $S$ is a set of $v \in \mathbb{R}_{>-M} \backslash \mathbb{Z}_{\triangleleft}$ for some $M \in \mathbb{Z}_{>-1}$.

We then express $u(t)$ as follows;

$$
u(t)=\sum_{v \in S} u_{v-1} g_{v}(t) .
$$

In [3] [4], we take up Kummer's differential equation as an example, which is

$$
t \cdot \frac{\mathrm{d}^{2}}{\mathrm{~d} t^{2}} u(t)+(c-t) \cdot \frac{\mathrm{d}}{\mathrm{d} t} u(t)-a \cdot u(t)=0, \quad t>0,
$$

where $a, c \in \mathbb{R}$ are constants. If $c \notin \mathbb{Z}$, one of the solutions given in [9] [10] is

$$
{ }_{1} F_{1}(a ; c ; t):=\sum_{n=0}^{\infty} \frac{(a)_{n}}{(c)_{n} n !} t^{n},
$$

where $(a)_{n}=\prod_{k=0}^{n-1}(a+k)$ for $a \in \mathbb{R}$ and $n \in \mathbb{Z}_{>0}$, and $(a)_{0}=1$. The other solution is

$$
t^{1-c} \cdot{ }_{1} F_{1}(a-c+1 ; 2-c ; t) \text {. }
$$

In [3], if $c<2$, we obtain both of the solutions. But when $c \geq 2$, (1.7) does not satisfy Condition 1 and we could not get it in [3]. In [4], we always obtain both of the solutions. In [1] [2], Yosida obtained only the solution (1.7).

We now study the solution of a differential equation with the aid of Laplace transform. Then it is required that there exists the Laplace transform of the function $u(t)$ to be determined.

When we consider the Laplace transform of a function $f(t)$ which is locally integrable on $\mathbb{R}_{>0}$, we assume the following condition.

Condition 3. There exists some $\lambda \in \mathbb{R}_{>0}$ such that $f(t) \mathrm{e}^{-\lambda t} \rightarrow 0$ as $t \rightarrow \infty$.

Let $f(t)$ be locally integrable on $\mathbb{R}_{>0}$ and satisfy Condition 3 , and the integral $\int_{0}^{1} f(t) \mathrm{d} t$ converge. We 
then denote its Laplace transform by $\tilde{f}(s)=\mathcal{L}[f(t)]$, so that

$$
\tilde{f}(s)=\mathcal{L}[f(t)]:=\int_{0}^{\infty} f(t) \mathrm{e}^{-s t} \mathrm{~d} t .
$$

The Laplace transform, $\tilde{g}_{v}(s)$, of $g_{v}(t)$ for $v \in \mathbb{R}_{>0}$ is then given by

$$
\tilde{g}_{v}(s)=s^{-v} \text {. }
$$

Let $u(t)$ expressed by (1.3) satisfy Condition 3, and let its Laplace transform $\tilde{u}(s)$ be given by

$$
\tilde{u}(s)=\sum_{v \in S_{1}} u_{v-1} s^{-v} .
$$

Then we can show that we are able to solve the problems solved in [3], with the aid of Laplace transform.

When $u(t)$ satisfies Conditions 2 and 3, Laplace transform is not applicable.

In [4], we adopted an analytic continuation of Riemann-Liouville fractional derivative, by which we could solve the differential equation assuming Condition 2. The analytic continuation is achieved with the aid of Pochhammer's contour, which is used in the analytic continuation of the beta function.

We now introduce the analytic continuation of Laplace transform with the aid of Hankel's contour, which is used in the analytic continuation of the gamma function. We then show that (1.9) is valid for $v \in \mathbb{R} \backslash \mathbb{Z}<$, and that if $u(t)$ expressed by (1.4) satisfies Condition 3, and its analytic continuation of Laplace transform of $u(t)$, which we denote by $\hat{u}(s)$, is given by

$$
\hat{u}(s)=\sum_{v \in S} u_{v-1} s^{-v},
$$

then we can solve the problems solved in [4], with the aid of the analytic continuation of Laplace transform.

In Section 2, we prepare the definition of analytic continuations of Riemann-Liouville fractional derivative and of Laplace transform, and then explain how the equation for the function $u(t)$ and its fractional derivative in (1.1) are converted into the corresponding equation for the analytic continuation of Laplace transform, $\hat{u}(s)$, of $u(t)$, and also how $\hat{u}(s)$ is converted back into $u(t)$. After these preparations, a recipe is given to be used in solving the fractional differential Equation (1.1) with the aid of the analytic continuation of Laplace transform in Section 3. In this recipe, the solution is obtained only when $a_{2} \neq 0$ and $b_{2}=0$. When $\sigma=\frac{1}{2}, b_{1}=0$ is also required. An explanation of this fact is given in Appendices C and D of [3]. In Section 4, we apply the recipe to (1.1) where $\sigma=1$ and $a_{0}=0$, of which special one is Kummer's differential equation. In Section 5, we apply the recipe to the fractional differential equation with $\sigma=\frac{1}{2}$, assuming $a_{0}=0$.

In Section 6, comments are given on the relation of the present method with the preceding one developed in [4], and on the application of the analytic continuation of Laplace transform to the differential equations with constant coefficients.

\section{Formulas}

Lemma 1. Let $g_{v}(t)$ be defined by (1.2). Then for $v \in \mathbb{R} \backslash \mathbb{Z}_{<1}$,

$$
t \cdot g_{v}(t)=v \cdot g_{v+1}(t), \quad t>0 .
$$

Proof. By (1.2), for $v \notin \mathbb{Z}_{<1}, t \cdot g_{v}(t)=\frac{1}{\Gamma(v)} t^{v}=\frac{v}{\Gamma(v+1)} t^{v}=v \cdot g_{v+1}(t)$.

\subsection{Analytic Continuation of Riemann-Liouville Fractional Derivative}

Let a function $f(t)$ be locally integrable on $\mathbb{R}_{>b}$ for $b \in \mathbb{R}$, and let $\int_{b}^{b+1} f(t) \mathrm{d} t$ exist. We then define the Riemann-Liouville fractional integral, ${ }_{b} \tilde{D}_{R}^{-\lambda} f(t)$, of order $\lambda \in \mathbb{R}_{>0}$ by

$$
{ }_{b} \tilde{D}_{R}^{-\lambda} f(t)=\frac{1}{\Gamma(\lambda)} \int_{b}^{t}(t-x)^{\lambda-1} f(x) \mathrm{d} x, \quad t>b .
$$


We then define the Riemann-Liouville fractional derivative, ${ }_{b} D_{R}^{\beta} f(t)$, of order $\beta \in \mathbb{R}$, by

$$
{ }_{b} D_{R}^{\beta} f(t)=\frac{\mathrm{d}^{N}}{\mathrm{~d} t^{N}}\left[{ }_{b} \tilde{D}_{R}^{\beta-N} f(t)\right], \quad t>b,
$$

if it exists, where $N=\max \{\lceil\beta\rceil, 0\}$, and ${ }_{b} \tilde{D}_{R}^{0} f(t)=f(t)$ for $t>b$.

For $v, t>0$ and $b=0$, we have

$$
{ }_{0} D_{R}^{\beta} g_{v}(t)= \begin{cases}g_{v-\beta}(t), & v-\beta \in \mathbb{R} \backslash \mathbb{Z}_{<1}, \\ 0, & v-\beta \in \mathbb{Z}_{<1} .\end{cases}
$$

If we assume that $\beta=-\lambda$ takes a complex value, ${ }_{0} \tilde{D}_{R}^{\beta} g_{v}(t)$ by definition (2.2) is analytic function of $\beta$ in the domain $\operatorname{Re} \beta<0$, and ${ }_{0} D_{R}^{\beta} g_{v}(t)$ defined by (2.3) is its analytic continuation to the whole complex plane. If we assume that $v$ also takes a complex value, ${ }_{0} D_{R}^{\beta} g_{v}(t)$ defined by (2.3) is an analytic function of $v$ in the domain $\operatorname{Re} v>0$. The analytic continuation as a function of $v$ was also studied. The argument is concluded that (2.4) should apply for the analytic continuation via Pochhammer's contour, even in $\operatorname{Re} v \leq 0$ except at the points where $v \in \mathbb{Z}_{<1}$; see [8].

We now adopt this analytic continuation of ${ }_{0} D_{R}^{\beta} g_{v}(t)$ to represent ${ }_{0} D_{R}^{\beta} g_{v}(t)$, and hence we accept the following lemma.

Lemma 2. Formula (2.4) holds for every $\beta \in \mathbb{R}, \quad v \in \mathbb{R} \backslash \mathbb{Z}_{<1}$.

By (1.4) and (2.4), we have

$$
{ }_{0} D_{R}^{\beta} u(t)=\sum_{v \in S, v-\beta \notin \mathbb{Z}_{\triangleleft}} u_{v-1} g_{v-\beta}(t) .
$$

For $u(t)$ defined by (1.4), we note that ${ }_{0} D_{R}^{-M} u(t)$ is locally integrable on $\mathbb{R}_{>0}$.

\subsection{Analytic Continuation of Laplace Transform}

The gamma function $\Gamma(z)$ for $z \in \mathbb{C}$ satisfying $\operatorname{Re} z>0$, is defined by Euler's second integral:

$$
\Gamma(z)=\int_{0}^{\infty} t^{z-1} \mathrm{e}^{-t} \mathrm{~d} t .
$$

The analytic continuation of $\Gamma(z)$ for $z \in \mathbb{C}$ is given by Hankel's formula:

$$
\Gamma(z)=\mathrm{e}^{-i \pi z} \frac{1}{2 i \sin \pi z} \int_{C_{H}} \zeta^{z-1} \mathrm{e}^{-\zeta} \mathrm{d} \zeta,
$$

where $C_{H}$ is Hankel's contour shown in Figure 1(a).

We now define an integral transform $\hat{f}(s)=\mathcal{L}_{H}[f(t)]$ of a function $f(t)$ which satisfies the following condition.

Condition 4. $f(z)$ is expressed as $f(z)=z^{\gamma-1} f_{1}(z)$ on a neighborhood of $\mathbb{R}_{>0}$, for $0 \leq \arg z<2 \pi$, where $\gamma \in \mathbb{C} \backslash \mathbb{Z}_{<1}$, and $f_{1}(z)$ is analytic on the neighborhood of $\mathbb{R}_{>0}$.

Let $f(t)$ satisfy Conditions 3 and 4 . Then we define $\hat{f}(s)$ for $\gamma \in \mathbb{C} \backslash \mathbb{Z}_{<1}$, by $\hat{f}(s)=\mathcal{L}_{H}[f(t)]$, where

$$
\mathcal{L}_{H}[f(t)]=\mathrm{e}^{-i \pi \gamma} \frac{1}{2 i \sin \pi \gamma} \int_{C_{H}} f(\zeta) \mathrm{e}^{-s \zeta} \mathrm{d} \zeta,
$$

for $\gamma \in \mathbb{C} \backslash \mathbb{Z}$, and

$$
\mathcal{L}_{H}[f(t)]=\lim _{\gamma_{i} \rightarrow n} \mathcal{L}_{H}\left[t^{\gamma_{i}-1} \cdot f_{1}(t)\right]
$$

for $\gamma=n \in \mathbb{Z}_{>0}, \gamma_{i} \in \mathbb{C} \backslash \mathbb{Z}$.

Lemma 3. Let $f(t)$ satisfy Condition 4. Then $\hat{f}(s)$ defined by (2.8) is an analytic continuation of $\tilde{f}(s)$, which is defined by (1.8) for $\operatorname{Re} \gamma>0$, as a function of $\gamma$.

Proof. The equality $\hat{f}(s)=\tilde{f}(s)$ when $\operatorname{Re} \gamma>0$ is proved in the same way as the equality of $\Gamma(z)$ given by (2.6) and by (2.7) for Re $z>0$; see e.g. ([11], Section 12.22). The analyticity of $\hat{f}(s)$ and of $\tilde{f}(s)$ is proved as in ([11], Sections 5.31, 5.32).

Lemma 4. For $v \in \mathbb{R} \backslash \mathbb{Z}_{<1}$, 


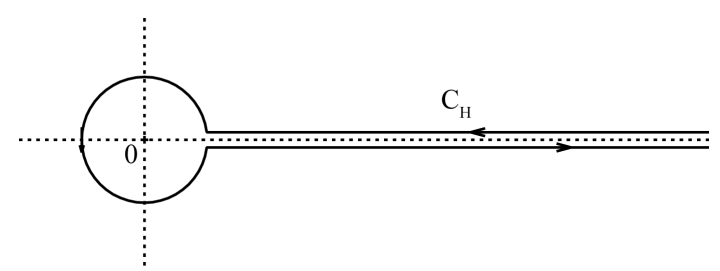

(a)

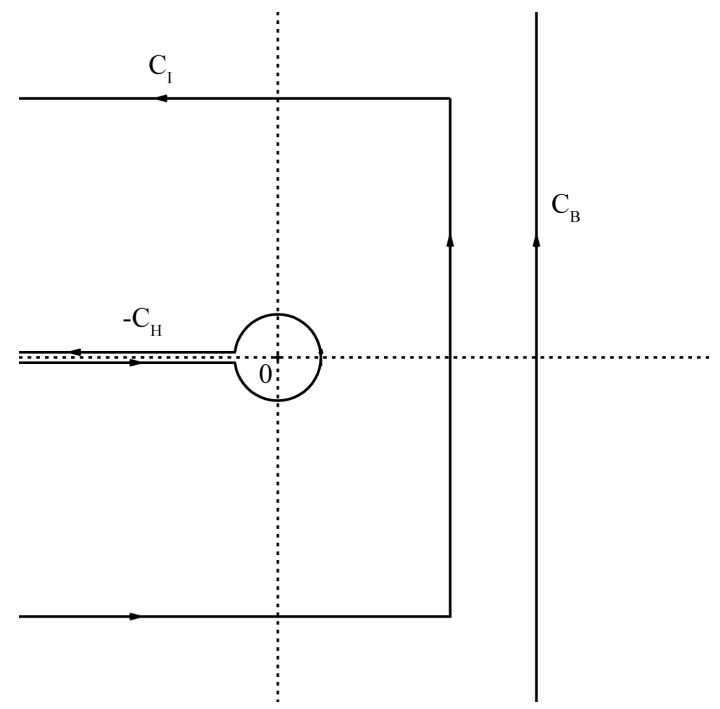

(b)

Figure 1. (a) Hankel's contour $C_{H}$, and (b) contours $C_{I},-C_{H}$ and $C_{B}$ which appear in (2.12), (2.15) and (2.16).

$$
\begin{gathered}
\hat{g}_{v}(s)=s^{-v}, \\
\mathcal{L}_{H}\left[t \cdot g_{v}(t)\right]=-\frac{\mathrm{d}}{\mathrm{d} s} \hat{g}_{v}(s), \\
g_{v}(t)=\frac{1}{2 \pi i} \int_{-C_{H}} s^{-v} \mathrm{e}^{s t} \mathrm{~d} s=\frac{1}{2 \pi i} \int_{C_{I}} s^{-v} \mathrm{e}^{s t} \mathrm{~d} s,
\end{gathered}
$$

where $-C_{H}$ and $C_{I}$ are two of the contours shown in Figure 1(b).

Proof. Formula (2.8) for $f(t)=g_{\gamma}(t)$ gives

$$
\hat{g}_{v}(s)=\mathrm{e}^{-i \pi \gamma} \frac{1}{2 i \sin \pi \gamma} \int_{C_{H}} \frac{1}{\Gamma(\gamma)} \zeta^{\gamma-1} \mathrm{e}^{-s \zeta} \mathrm{d} \zeta=s^{-\gamma},
$$

for $\gamma \in \mathbb{C} \backslash \mathbb{Z}$. The last equality in (2.13) is due to (1.9). By using (2.1) and (2.10), the lefthand side of (2.11) is expressed as $\mathcal{L}_{H}\left[v \cdot g_{v+1}(t)\right]=v \cdot s^{-v-1}=-\frac{\mathrm{d}}{\mathrm{d} s} s^{-v}=-\frac{\mathrm{d}}{\mathrm{d} s} \hat{g}_{v}(s)$. By replacing $s, \gamma$ and $\zeta$ in (2.13), by $t$, $-v+1$ and $\mathrm{e}^{-i \pi} s$, respectively, we obtain the first equality of (2.12) for $v \notin \mathbb{Z}$, with the aid of the formula $\frac{\pi}{\sin \pi z}=\Gamma(z) \Gamma(1-z)$. The equality for $v \in \mathbb{Z}_{>0}$ is obtained by continuity.

Theorem 1. Let $f(t)$ satisfy Conditions 3 and 4 for $\gamma>0$, and $u(t)$ be expressed as

$$
u(t)=\sum_{v \in S_{0}} u_{v-1} g_{v}(t)+f(t)
$$

where $S_{0}$ is a finite set of $v \in \mathbb{R} \backslash \mathbb{Z}_{<1}$. Then the Laplace inversion formula is given by the contour integral: 


$$
u(t)=\mathcal{L}_{H}^{-1}[\hat{u}(s)]:=\frac{1}{2 \pi i} \int_{C_{I}} \hat{u}(s) \mathrm{e}^{s t} \mathrm{~d} s,
$$

where $C_{I}$ is a contour shown in Figure 1(b). Here it is assumed that $\hat{f}(s)$ is analytic to the right of the vertical line on $C_{\mathrm{I}}$, and is so above and below the upper and lower horizontal lines, respectively, on $C_{I}$.

Proof. For $f(t)$, the usual Laplace inversion formula applies, so that

$$
f(t)=\frac{1}{2 \pi i} \int_{C_{B}} \hat{f}(s) \mathrm{e}^{s t} \mathrm{~d} s=\frac{1}{2 \pi i} \int_{C_{I}} \hat{f}(s) \mathrm{e}^{s t} \mathrm{~d} s,
$$

where $C_{B}$ is a contour shown in Figure 1(b). Here it is assumed that $\hat{f}(s)$ is analytic to the right of the contour $C_{B}$. By using this with (2.10) and (2.12), we confirm (2.15).

Lemma 5. Let $f(t)$ satisfy Conditions 3 and 4 with an entire function $f_{1}(t)$. Then

$$
\hat{f}(s)=s^{-\gamma} \sum_{n=0}^{\infty} f_{1}^{(n)}(0) \frac{\Gamma(n+\gamma)}{n !} s^{-n} .
$$

Lemma 6. Let $\hat{u}(s)$ be expressed in the form of (1.11). Then the Laplace inversion $u(t)$ is given by (1.4), provided that the obtained $u(t)$ satisfies the conditions for $f(t)$ in Lemma 5 , or it is a linear combination of such functions.

Lemma 7. Let $u(t)$ satisfy the conditions for $f(t)$ in Lemma 5. Then

$$
\begin{gathered}
\mathcal{L}_{H}\left[{ }_{0} D_{R}^{\beta} u(t)\right]=\sum_{v \in S, v-\beta \notin \mathbb{Z}_{<}} u_{v-1} \hat{g}_{v-\beta}(s)=\sum_{v \in S, v-\beta \notin \mathbb{Z}_{<1}} u_{v-1} s^{-v+\beta} \\
=s^{\beta} \hat{u}(s)-\sum_{k \in \mathbb{Z}_{>-1}, \beta-k \in S} u_{\beta-k-1} s^{k}, \\
\mathcal{L}_{H}\left[t \cdot{ }_{0} D_{R}^{\beta} u(t)\right]=-\frac{\mathrm{d}}{\mathrm{d} s} \mathcal{L}_{H}\left[{ }_{0} D_{R}^{\beta} u(t)\right] .
\end{gathered}
$$

Proof. By using (2.5) and Lemma 4, we obtain these results.

\section{Recipe of Solving Laplace's Differential Equation and Fractional Differential Equation of That Type}

We now express the differential Equation (1.1) to be solved, as follows:

$$
\sum_{l=0}^{m}\left(a_{l} t+b_{l}\right) \cdot{ }_{0} D_{R}^{l \sigma} u(t)=f(t), \quad t>0,
$$

where $\sigma=\frac{1}{2}$ or $\sigma=1$, and $m=2$. In Sections 4 and 5 , we study this differential equation for $\sigma=1$ and this fractional differential equation for $\sigma=\frac{1}{2}$, respectively.

We now apply the integral transform $\mathcal{L}_{H}$ to (3.1). By using (2.18) and (2.19), we then obtain

$$
-A(s) \frac{\mathrm{d}}{\mathrm{d} s} \hat{u}(s)+B(s) \hat{u}(s)=\hat{f}(s)+\hat{v}(s) \text {, }
$$

where

$$
\begin{gathered}
A(s)=\sum_{l=0}^{m} a_{l} \cdot s^{l \sigma}, \\
B(s)=\sum_{l=0}^{m}\left(-l \sigma \cdot a_{l} \cdot s^{l \sigma-1}+b_{l} \cdot s^{l \sigma}\right), \\
\hat{v}(s)=\sum_{l=1}^{m}\left[-a_{l} \sum_{k \in \mathbb{Z}^{*}} k \cdot u_{l \sigma-k-1} \cdot s^{k-1}+b_{l} \sum_{k \in \mathbb{Z}^{*}} u_{l \sigma-k-1} \cdot s^{k}\right] .
\end{gathered}
$$


Here $\mathbb{Z}^{*}:=\left\{k \in \mathbb{Z}_{>-1} \mid \sigma-k \notin \mathbb{Z}_{<1}\right\}$.

Lemma 8. The complementary solution (C-solution) of Equation (3.2) is given by $\hat{u}(s)=C_{1} \cdot \hat{\phi}(s)$, where $C_{1}$ is an arbitrary constant and

$$
\hat{\phi}(s)=C_{2} \cdot \exp \left(\int^{s} \frac{B(\xi)}{A(\xi)} \mathrm{d} \xi\right),
$$

where the integral is the indefinite integral and $C_{2}$ is any constant.

Lemma 9. Let $\hat{\phi}(s)$ be the C-solution of (3.2), and $\hat{u}_{v}^{*}(s)$ be the particular solution (P-solution) of (3.2), when the inhomogeneous term is $s^{-v}$ for $v \in \mathbb{R}$. Then

$$
\hat{u}_{v}^{*}(s)=-\hat{\phi}(s) \int^{s} \frac{\xi^{-v}}{A(\xi) \hat{\phi}(\xi)} \mathrm{d} \xi+C_{3} \cdot \hat{\phi}(s),
$$

where $C_{3}$ is any constant.

Since $f(t)$ in (3.1) satisfies Condition 2 and $\hat{v}(s)$ is given by (3.4), the P-solution $\hat{u}(s)$ of (3.2) is expressed as a linear combination of $\hat{u}_{v}^{*}(s)$ for $v \in \mathbb{R}_{>-M}$ for $M \in \mathbb{Z}_{>-1}$, and of $\hat{u}_{-k}^{*}(s)$ for $k \in \mathbb{Z}_{>-1}$, respectively.

The solution $\hat{u}(s)$ of (3.2) is converted to a solution $u(t)$ of (3.1) for $t>0$, with the aid of Lemma 6 .

\section{Laplace's and Kummer's Differential Equations}

We now consider the case of $\sigma=1, m=2, a_{2} \neq 0, a_{1} \neq 0$, and $a_{0}=b_{2}=0$. Then (3.1) reduces to

$$
a_{2} t \cdot \frac{\mathrm{d}^{2}}{\mathrm{~d} t^{2}} u(t)+\left(a_{1} t+b_{1}\right) \cdot \frac{\mathrm{d}}{\mathrm{d} t} u(t)+b_{0} \cdot u(t)=f(t), \quad t>0
$$

By (3.3) and (3.4), $A(s), B(s)$ and $\hat{v}(s)$ are

$$
\begin{gathered}
A(s)=a_{2} s^{2}+a_{1} s=a_{2} s(s+\alpha), \quad \alpha=\frac{a_{1}}{a_{2}}, \\
B(s)=\left(b_{1}-2 a_{2}\right) s+b_{0}-a_{1}, \\
\hat{v}(s)=\left(-a_{2}+b_{1}\right) u_{0} .
\end{gathered}
$$

\subsection{Complementary Solution of (3.2) and (4.1)}

In order to obtain the C-solution $\hat{\phi}(s)$ of (3.2) by using (3.5), we express $B(s) / A(s)$ as follows:

$$
\frac{B(s)}{A(s)}=\frac{\gamma_{1}}{s}+\frac{\gamma_{2}}{s+\alpha},
$$

where

$$
\gamma_{1}+\gamma_{2}=\frac{b_{1}}{a_{2}}-2, \quad \gamma_{1}=\frac{b_{0}}{a_{1}}-1, \quad \gamma_{2}=\frac{b_{1}}{a_{2}}-\frac{b_{0}}{a_{1}}-1
$$

By using (3.5), we obtain

$$
\hat{\phi}(s)=s^{\gamma_{1}}(s+\alpha)^{\gamma_{2}}=s^{\gamma_{1}+\gamma_{2}}\left(1+\alpha s^{-1}\right)^{\gamma_{2}}=s^{\gamma_{1}+\gamma_{2}} \sum_{n=0}^{\infty}\left(\begin{array}{c}
\gamma_{2} \\
n
\end{array}\right) \alpha^{n} s^{-n},
$$

in the form of (2.17) or (1.11), where $\left(\begin{array}{l}\gamma \\ n\end{array}\right)=\frac{(-1)^{n}(-\gamma)_{n}}{n !}$ for $\gamma \in \mathbb{R}$ and $n \in \mathbb{Z}_{>-1}$ are the binomial coefficients.

If $\gamma_{1}+\gamma_{2} \notin \mathbb{Z}_{>-1}$, we obtain a C-solution of (4.1), by using Lemma 6: 


$$
\begin{aligned}
u(t) & =C_{1} \cdot \phi(t)=C_{1} \cdot t^{-\gamma_{1}-\gamma_{2}-1} \sum_{n=0}^{\infty}\left(\begin{array}{c}
\gamma_{2} \\
n
\end{array}\right) \alpha^{n} \frac{1}{\Gamma\left(n-\gamma_{1}-\gamma_{2}\right)} t^{n} \\
& =C_{1} \cdot \frac{1}{\Gamma\left(-\gamma_{1}-\gamma_{2}\right)} t^{-\gamma_{1}-\gamma_{2}-1} \cdot{ }_{1} F_{1}\left(-\gamma_{2} ;-\gamma_{1}-\gamma_{2} ;-\alpha t\right) .
\end{aligned}
$$

Remark 1. In Introduction, Kummer's differential equation is given by (1.5). It is equal to (4.1) for $a_{2}=1$, $a_{1}=-1, b_{1}=c$ and $b_{0}=-a$. In this case,

$$
\gamma_{2}=c-a-1, \quad \gamma_{1}=a-1, \quad \gamma_{1}+\gamma_{2}=c-2, \quad \alpha=-1 .
$$

We then confirm that the expression (4.8) for $c \notin \mathbb{Z}_{>1}$ agrees with (1.7), which is one of the $C$-solutions of Kummer's differential equation given in [9] [10].

\subsection{Particular Solution of (3.2)}

We now obtain the P-solution of (3.2), when the inhomogeneous term is equal to $s^{-v}$ for $v \in \mathbb{R}$.

When the C-solution of (3.2) is $\hat{\phi}(s)$, the P-solution of (3.2) is given by (3.6). By using (4.2) and (4.6), the following result is obtained in [3]:

$$
\begin{aligned}
\hat{u}_{v}^{*}(s) & =-s^{\gamma_{1}}(s+\alpha)^{\gamma_{2}} \int^{s} \frac{\xi^{-v}}{a_{2} \xi^{1+\gamma_{1}}(\xi+\alpha)^{1+\gamma_{2}}} \mathrm{~d} \xi+C_{3} \cdot \hat{\phi}(s) \\
& =\frac{1}{a_{2}} s^{-1-\nu} \sum_{n=0}^{\infty}{ }_{n} C_{\gamma_{2}, \gamma_{1}+1+\nu}^{*} \cdot \alpha^{n} s^{-n},
\end{aligned}
$$

where

$$
{ }_{n} C_{p_{1}, p_{2}}^{*}=\sum_{k=0}^{n}\left(\begin{array}{c}
p_{1} \\
k
\end{array}\right)\left(\begin{array}{c}
-p_{1}-1 \\
n-k
\end{array}\right) \frac{1}{n-k+p_{1}+p_{2}} .
$$

Lemma 10. When $p_{1}+p_{2} \notin \mathbb{Z}_{<1},{ }_{n} C_{p_{1}, p_{2}}^{*}$ defined by (4.11) is expressed as

$$
{ }_{n} C_{p_{1}, p_{2}}^{*}=\frac{(-1)^{n}}{p_{1}+p_{2}} \cdot{ }_{n} C_{p_{1}, p_{2}}^{\dagger},
$$

where

$$
{ }_{n} C_{p_{1}, p_{2}}^{\dagger}=\frac{\left(p_{2}\right)_{n}}{\left(1+p_{1}+p_{2}\right)_{n}} .
$$

This lemma is proved in [3]. In fact, (4.11) is the partial fraction expansion of ${ }_{n} C_{p_{1}, p_{2}}^{*}$ given by (4.12) as a function of $p_{2}$.

Applying Lemma 6 to (4.10), and using (4.12), we obtain

Theorem 2. Let $v \in \mathbb{R} \backslash \mathbb{Z}_{<0}, 1+v+\gamma_{1}+\gamma_{2} \notin \mathbb{Z}_{<1}$, and let $f(t)=g_{v}(t)$ for $t>0$. Then we have a P-solution $u_{v}^{*}(t)$ of (4.1), given by

$$
u_{v}^{*}(t)=\frac{1}{a_{2} \cdot\left(1+v+\gamma_{1}+\gamma_{2}\right)} u_{v}^{\dagger}(t),
$$

where

$$
\begin{aligned}
u_{v}^{\dagger}(t) & =t^{\nu} \sum_{n=0}^{\infty} \frac{\left(v+1+\gamma_{1}\right)_{n}}{\left(2+v+\gamma_{1}+\gamma_{2}\right)_{n} \cdot \Gamma(v+n+1)}(-\alpha t)^{n} \\
& =\frac{1}{\Gamma(v+1)} t^{\nu} \cdot{ }_{2} F_{2}\left(v+1+\gamma_{1}, 1 ; 2+v+\gamma_{1}+\gamma_{2}, v+1 ;-\alpha t\right) .
\end{aligned}
$$

Here ${ }_{2} F_{2}\left(a_{1}, a_{2} ; b_{1}, b_{2} ; x\right)=\sum_{n=0}^{\infty} \frac{\left(a_{1}\right)_{n}\left(a_{2}\right)_{n}}{\left(b_{1}\right)_{n}\left(b_{2}\right)_{n} n !} x^{n}$. 


\subsection{Complementary Solution of (4.1)}

By (4.3) and (4.5), $\hat{v}(s)=a_{2} \cdot\left(1+\gamma_{1}+\gamma_{2}\right) \cdot u_{0}$. When $\hat{f}(s)=0$ and $\hat{v}(s) \neq 0$, the P-solution of (3.2) is given by

$$
\hat{u}(s)=a_{2} \cdot\left(1+\gamma_{1}+\gamma_{2}\right) \cdot u_{0} \cdot \hat{u}_{0}^{*}(s) .
$$

By using (4.14) for $v=0$, if $1+\gamma_{1}+\gamma_{2} \notin \mathbb{Z}_{<1}$, we obtain a C-solution of (4.1):

$$
u(t)=u_{0} \cdot u_{0}^{\dagger}(t)=u_{0} \cdot{ }_{1} F_{1}\left(1+\gamma_{1} ; 2+\gamma_{1}+\gamma_{2} ;-\alpha t\right) .
$$

In Section 4.1, we have (4.7), that is another C-solution of (4.1). If we compare (4.7) with (4.15), when $\gamma_{1}+\gamma_{2} \notin \mathbb{Z}_{>-1}$, it can be expressed as

$$
C_{1} \cdot \phi(t)=C_{1} \cdot u_{-\gamma_{1}-\gamma_{2}-1}^{\dagger}(t)
$$

Proposition 1. When $\gamma_{1}+\gamma_{2} \notin \mathbb{Z}$, the complementary solution of (4.1) is given by the sum of the righthand sides of (4.8) and of (4.18), which are equal to $C_{1} \cdot u_{-\gamma_{1}-\gamma_{2}-1}^{\dagger}(t)$ and $u_{0} \cdot u_{0}^{\dagger}(t)$, respectively.

Remark 2. As stated in Remark 1, for Kummer's differential equation, $\gamma_{1}$ and $\gamma_{2}$ are given in (4.9), and

$$
1+\gamma_{1}=a, \quad 2+\gamma_{1}+\gamma_{2}=c, \quad \alpha=-1 .
$$

We then confirm that if $c \notin \mathbb{Z}$, the set of (4.8) and (4.18) agrees with the set of (1.6) and (1.7).

\section{Solution of Fractional Differential Equation (3.1) for $\sigma=1 / 2$}

In this section, we consider the case of $\sigma=\frac{1}{2}, m=2, a_{2} \neq 0, a_{1} \neq 0, a_{0}=b_{2}=b_{1}=0$ and $b_{0} \neq 0$. Then the Equation (3.1) to be solved is

$$
a_{2} t \cdot{ }_{0} D_{R} u(t)+a_{1} t \cdot{ }_{0} D_{R}^{1 / 2} u(t)+b_{0} \cdot u(t)=f(t), \quad t>0 .
$$

Now (3.3) and (3.4) are expressed as

$$
\begin{gathered}
A(s)=a_{2} s+a_{1} s^{1 / 2}=a_{2} s^{1 / 2}\left(s^{1 / 2}+\alpha\right), \quad \alpha=\frac{a_{1}}{a_{2}}, \\
B(s)=b_{0}-a_{2}-\frac{1}{2} a_{1} s^{-1 / 2}, \\
\hat{v}(s)=-a_{1} \sum_{k=0}^{M}(k+1) u_{1 / 2-k-2} \cdot s^{k} .
\end{gathered}
$$

\subsection{Complementary Solution of (3.2)}

By using (5.2), $B(s) / A(s)$ is expressed as

$$
\frac{B(s)}{A(s)}=s^{-1 / 2}\left(\frac{\gamma_{1}}{s^{1 / 2}}+\frac{\gamma_{2}}{s^{1 / 2}+\alpha}\right)
$$

where

$$
\gamma_{1}+\gamma_{2}=\frac{b_{0}}{a_{2}}-1, \quad \gamma_{1}=-\frac{1}{2}, \quad \gamma_{2}=\frac{b_{0}}{a_{2}}-\frac{1}{2} .
$$

By (3.5), the C-solution $\hat{\phi}(s)$ of (3.2) is given by

$$
\hat{\phi}(s)=s^{\gamma_{1}}\left(s^{1 / 2}+\alpha\right)^{2 \gamma_{2}}=s^{\gamma_{1}+\gamma_{2}}\left(1+\alpha s^{-1 / 2}\right)^{2 \gamma_{2}}=s^{\gamma_{1}+\gamma_{2}} \sum_{n=0}^{\infty}\left(\begin{array}{c}
2 \gamma_{2} \\
n
\end{array}\right) \alpha^{n} s^{-n / 2} .
$$

If $2\left(\gamma_{1}+\gamma_{2}\right) \notin \mathbb{Z}_{>-1}$, by applying Lemma 6 to this, we obtain the C-solution of (5.1): 


$$
u(t)=C_{1} \cdot \phi(t)=C_{1} \cdot t^{-\gamma_{1}-\gamma_{2}-1} \sum_{n=0}^{\infty}\left(\begin{array}{c}
2 \gamma_{2} \\
n
\end{array}\right) \frac{1}{\Gamma\left(\frac{n}{2}-\gamma_{1}-\gamma_{2}\right)} \alpha^{n} t^{n / 2}
$$

\subsection{Particular Solution of (3.2) and (5.1)}

By using the expressions of $A(s)$ and $\hat{\phi}(s)$ given by (5.2) and (5.6) in (3.6), we obtain the P-solution of (3.2), when the inhomogeneous term is $x^{-v}$ for $v \in \mathbb{R}$ satisfying $2\left(v+\gamma_{1}+\gamma_{2}\right) \notin \mathbb{Z}_{<1}$ :

$$
\begin{aligned}
\hat{u}_{v}^{*}(s) & =-s^{\gamma_{1}}\left(s^{1 / 2}+\alpha\right)^{2 \gamma_{2}} \int^{s} \frac{\xi^{-v}}{a_{2} \xi^{1 / 2+\gamma_{1}}\left(\xi^{1 / 2}+\alpha\right)^{1+2 \gamma_{2}}} \mathrm{~d} \xi+C_{3} \cdot \hat{\phi}(s) \\
& =\frac{2}{a_{2}} s^{-v} \sum_{n=0}^{\infty}{ }_{n} C_{2 \gamma_{2}, 2 \gamma_{1}+2 v}^{*} \cdot \alpha^{n} s^{-n / 2},
\end{aligned}
$$

where ${ }_{n} C_{2 \gamma_{2}, 2 \gamma_{1}+2 v}^{*}$ is defined by (4.11) and is given by (4.12).

By applying Lemma 6 to this, we obtain the following theorem.

Theorem 3. Let $v \in \mathbb{R}, 2 v \notin \mathbb{Z}_{<1}, 2\left(v+\gamma_{1}+\gamma_{2}\right) \notin \mathbb{Z}_{<1}$, and let $f(t)=g_{v}(t)$. Then we have a P-solution $u_{v}^{*}(t)$ of (5.1), given by

where

$$
u_{v}^{*}(t)=\frac{1}{a_{2} \cdot\left(v+\gamma_{1}+\gamma_{2}\right)} u_{v}^{\dagger}(t),
$$

$$
u_{v}^{\dagger}(t)=t^{v-1} \sum_{n=0}^{\infty} \frac{\left(2 v+2 \gamma_{1}\right)_{n}}{\left(1+2 v+2 \gamma_{1}+2 \gamma_{2}\right)_{n}} \cdot \frac{1}{\Gamma\left(\frac{n}{2}+v\right)}(-\alpha)^{n} t^{n / 2} .
$$

\subsection{Complementary Solution of (5.1)}

We obtain the solution $u_{v}^{*}(t)$ only for $2 v \notin \mathbb{Z}_{<1}$. When $\hat{v}(s)$ is given by (5.3) with nonzero values of $u_{1 / 2-k-2}$, Theorem 2 does not give a solution of (5.1). Hence $u(t)$ given by (5.7) is the only C-solution of (5.1).

If we compare (5.7) with (5.10), we obtain the following proposition.

Proposition 2. Let $2\left(\gamma_{1}+\gamma_{2}\right) \notin \mathbb{R}_{>-1}$. Then the $C$-solution of (5.1) is given by

$$
C_{1} \cdot \phi(t)=C_{1} \cdot u_{-\gamma_{1}-\gamma_{2}}^{\dagger}(t) \text {. }
$$

\section{Concluding Remarks}

\subsection{Solution with the Aid of Distribution Theory}

In [4], the solution of (3.1) is assumed to be expressed as (1.4). In distribution theory, the differential equation for the distribution $\tilde{u}(t)=u(t) H(t)$ is set up, where $H(t)=1$ for $t>0$ and $H(t)=0$ for $t \leq 0$. Then it is expressed as $\tilde{u}(t)=\hat{u}(D) \delta(t)=\sum_{v \in S} u_{v-1} D^{-v} \delta(t)$, where $D^{-v}$ is the derivative of order $-v$ in the space of $\mathcal{D}_{R}^{\prime}$ of distributions. In [4], after obtaining $\hat{u}(D), u(t)$ is obtained by using Neumann series expansion. In the present paper, $\hat{u}(s)$ is the analytic continuation of Laplace transform of $u(t)$. After obtaining $\hat{u}(s)$, we obtain $u(t)$ for $t>0$ by Laplace inversion.

The steps of solution in [4] and the present paper are closely related with each other, and one may use a favorite one. One difference is that Condition 3 is assumed in the present paper but is not required in [4].

\subsection{Solutions of Differential Equations with Constant Coefficients}

We now consider the differential equation given by (3.1), where $a_{2}=a_{1}=a_{0}=0$. Then assuming that the solution $u(t)$ and the inhomogeneous term $f(t)$ satisfy Conditions 2 and 3, we show that the analytic continuation of Laplace transform of that equation is given by (3.2) with $A(s)=0$. We then obtain the analytic continu- 
ation of Laplace transform of $u(t), \hat{u}(s)$. If it can be expressed as (1.11), then $u(t)$ is given by its Laplace inverse (1.4). If we take account of Section 6.1, we confirm that the results obtained in [7] are obtained by Laplace inversion.

\section{References}

[1] Yosida, K. (1983) The Algebraic Derivative and Laplace’s Differential Equation. Proceedings of Japan Academy, 59, 1-4. http://dx.doi.org/10.3792/pjaa.59.1

[2] Yosida, K. (1982) Operational Calculus. Springer-Verlag, New York, Chapter VII.

[3] Morita, T. and Sato, K. (2013) Remarks on the Solution of Laplace's Differential Equation and Fractional Differential Equation of That Type. Applied Mathematics, 4, 13-21. http://dx.doi.org/10.4236/am.2013.411A1003

[4] Morita, T. and Sato, K. (2013) Solution of Laplace's Differential Equation and Fractional Differential Equation of That Type. Applied Mathematics, 4, 26-36. http://dx.doi.org/10.4236/am.2013.411A1005

[5] Mikusiński, J. (1959) Operational Calculus. Pergamon Press, London.

[6] Morita, T. and Sato, K. (2006) Solution of Fractional Differential Equation in Terms of Distribution Theory. Interdisciplinary Information Sciences, 12, 71-83.

[7] Morita, T. and Sato, K. (2010) Neumann-Series Solution of Fractional Differential Equation. Interdisciplinary Information Sciences, 16, 127-137. http://dx.doi.org/10.4036/iis.2010.127

[8] Morita, T. and Sato, K. (2013) Liouville and Riemann-Liouville Fractional Derivatives via Contour Integrals. Fractional Calculus and Applied Analysis, 16, 630-653. http://dx.doi.org/10.2478/s13540-013-0040-9

[9] Abramowitz, M. and Stegun, I.A. (1972) Handbook of Mathematical Functions with Formulas, Graphs and Mathematical Tables. Dover Publ., Inc., New York, Chapter 13.

[10] Magnus, M. and Oberhettinger, F. (1949) Formulas and Theorems for the Functions of Mathematical Physics. Chelsea Publ. Co., New York, Chapter VI.

[11] Whittaker, E.T. and Watson, G.N. (1935) A Course of Modern Analysis. Cambridge U.P., Cambridge. 